\title{
EMBRYO RECONSTRUCTION BY TRANSPLANTATION OF THE DONOR INNER CELL MASS TO THE RECIPIENT BOVINE BLASTOCYST
}

\author{
ARIEF BOEDIONO \\ Laboratory of Embryology, Department of Anatomy, Physiology and Pharmacology, \\ Faculty of Veterinary Medicine, Bogor Agricultural University, Darmaga 16680Bogor, Indonesia
}

\begin{abstract}
In an attempt to produce the interspecies embryo transfer, this study was conducted to evaluate the efficacy of the production of reconstructed blastocyst by transferring the donor ICM into the recipient trophoblast. ICM cells were isolated from the donor blastocyst by immunosurgery method. Zona-free blastocysts were incubated in the medium (TCM-199) containing $20 \%$ of the heat-inactivated rabbit anti-bovine-serum. The embryo reconstruction was produced by three different methods. Recipient blastocyst was maintained on the holding pipette by gentle suction, with the ICM in a 9 o'clock position to have the possibility of developing incorporate ICMs (Method I), the ICM was in a 3 o'clock position to break the original ICM during injection (Method 2); cutting the original recipient ICM followed by insertion of the donor ICM (Method 3). Reconstructed blastocysts were then cultured overnight and examined morphologically according to the re-expansion of the reconstructed blastocyst with or without developed donor ICM. According to morphological observation in this study, $37.9 \%$ of the reconstructed blastocyst developed with the incorporation of two ICM originally from recipient and donor (Method I), 66.7\% of the reconstructed blastocysts developed with a single ICM (Method 2), and $80.0 \%$ of the reconstructed blastocyst developed from the ICM originally from donor ICM (Method 3). These results showed that the reconstructed blastocyst is better produced by cutting the original recipient ICM followed by the insertion of the donor ICM (Method 3).
\end{abstract}

Key words: embryo reconstruction, immunosurgery, ICM transfer, bovine.

\section{INTRODUCTION}

Embryo reconstruction for chimera production has been used in experiments oriented towards animal science such as the production of interspecies pregnancies in domestic animals (Fehilly et al. 1984a; Polzin et al. 1987). So far, chimeras have been obtained by the aggregation of the blastomeres (Brem et al. 1984; Boediono et al. 1993; Boediono et al. 1999) or by inner cell mass transplantation (Butler et al. 1987; Polzin et al. 1987; Picard et al. 1990). The aggregation of cell from embryos results in embryos with a more randomly distribution contribution of cells from each donor to the trophoblast and ICM. Fehilly et al. (1984b) in their production of interspecific sheep-goat chimeras produced one kid by the injection of a goat ICM into a sheep blastocyst and one lamb from the reciprocal injection. Rorie et al. (1994) produced two normal lambs after transferring nine reconstructed embryos into three recipient goats. The reconstructed embryos comprised trophoblast cells from the embryo recipient species (goat) and ICM cells from the embryo donor species (sheep). The present study was conducted to evaluate alternative procedures

Corresponding author: abl@cbn.net.id 
for production of the reconstructed embryo by transplantation of the donor inner cell mass to the recipient bovine blastocyst.

\section{MATERIALS AND METHOD}

\section{In vitro embryo production}

Embryos were produced by standard in vitro maturation, fertilization and culture procedures (Boediono et al. 2003). Bovine ovaries were collected from a nearby abattoir and transported $\left(32-35^{\circ} \mathrm{C}\right)$ to the laboratory in $0.9 \%$ physiological saline solution immediately following collection. Follicular oocytes were allowed to mature for $22 \mathrm{hr}$ at $38.5^{\circ} \mathrm{C}$ under $5 \% \mathrm{CO} 2$ in air. Frozen-thawed sperm was washed twice with $2.5 \mathrm{mM}$ caffeine in Brackett-Oliphant medium (B-O; Brackett and Oliphant 1975) without bovine serum albumin. Sperm concentration was adjusted to $5 \times 10^{6}$ spermatozoa per $\mathrm{ml}$ in B-O supplemented with $0.3 \%$ bovine serum albumin (BSA, Sigma) and $20 \wedge \mathrm{g} / \mathrm{ml}$ heparin (Shimizu, Japan). A100 ul aliquot of the sperm suspension was pre-incubated for $1 \mathrm{hr}$. In v//ra-matured oocytes were transferred into fertilization droplets for insemination (20-25 oocytes/droplet). After $18 \mathrm{hr}$ of sperm exposure, oocytes were washed and transferred to a polystyrene dish (4-well multidish; Nunclon, Roskilde, Denmark) containing TCM199 supplemented with $5 \%$ superovulated cow serum (Boediono et al. 1994), $5 \mathrm{ng} / \mathrm{ml}$ insulin (Wako, Osaka, Japan) and $50 \mathrm{ng} / \mathrm{ml}$ gentamicin sulfate for further development. Adherent cumulus cells surrounding the embryos were removed by repeated pipetting $48 \mathrm{~h}$ post-fertilization. The cumulus cells adhering to the surface of the culture dish were not disrupted, and embryos were cultured on this somatic cell monolayer. The culture medium was replaced with fresh medium $96 \mathrm{~h}$ post-fertilization.

\section{ICM isolation}

Blastocysts produced in vitro were used in this experiment. An immunosurgical method for ICM isolation was carried out by the similar method described by Softer and Knowles (1975) with modification. The zona pellucida was removed by a short incubation ( $<5 \mathrm{~min})$ in TCM-199 containing $0.5 \%$ pronase. For immunosurgery, the embryos were incubated for $1 \mathrm{~h}$ in the medium (TCM-199) containing $20 \%$ of the heat-inactivated rabbit anti-bovine-serum. They were then washed in three successive baths of TCM-199 + 20\% PCS and were transferred into TCM-199 containing 10\% of reconstituted guinea pig complement for 10 min followed by two successive washes in TCM-199 + 20\% PCS. Embryos were aspirated into a pipette of a diameter slightly larger than the ICM to remove any degenerating trophoblastic cell.

\section{Embryo reconstruction}

The manipulator was arranged with a beveled injection pipette with an outer diameter of approximately 40-50 $\mathrm{ll}$ on one side and an embryo holding pipette 
with an outer diameter of 150 urn on the other side (Method 1 and 2). The donor ICM was drawn into the injection pipette and held directly opposite the recipient blastocyst on the holding pipette. The embryo reconstruction was produced by three different methods. Recipient blastocyst was maintained on the holding pipette by gentle suction, with the ICM in a 9 o'clock position to have the possibility of developing an incorporate ICMs (Method 1, Figure 1A-B), the ICM was in a 3 o'clock position to break the original ICM during injection (Method 2, Figure 1C-D); cutting the original recipient ICM followed by insertion of the donor ICM (Method 3, Figure $1 \mathrm{E}-\mathrm{F})$. With a swift precise forward motion the tip of the injection pipette was introduced into the blastocoele and the ICM was injected. Reconstructed embryos were cultured overnight and then examined morphologically according to the re-expansion of blastocyst with the presence of original and donor ICMs (Method 1 and 2) and the re-expansion of the blastocyst with or without donor ICM (Method 3).

\section{RESULTS AND DISCUSSION}

Embryo reconstruction was performed by injection of a total of 78 ICM isolated from donor blastocysts into blastocoel recipient blastocysts. In an attempt to produce interspecies pregnancy, the development of the donor ICM with the original trophoblast is most important to produce a baby originating from donor ICM. According to morphological observation, 37.9\% of the reconstructed blastocyst produced by Method 1 developed from the incorporation of two ICM originally from recipient and donor, while $34.5 \%$ developed with the corporation of two ICM (Table 1). Using the same method, Fehilly et al. (1984b) in their production of inter

Table 1. Development of reconstructed blastocyst produced by ICM transfer followed by cultured in vitro.

\begin{tabular}{|c|c|c|c|c|c|}
\hline \multirow{3}{*}{$\begin{array}{l}\text { Reconstruction } \\
\text { Method }\end{array}$} & \multirow{3}{*}{$\begin{array}{l}\text { No. of } \\
\text { embryos }\end{array}$} & \multicolumn{3}{|c|}{ Reconstructed Blastocyst (\%) } & \multirow{3}{*}{$\begin{array}{c}\text { Degenerated } \\
(\%)\end{array}$} \\
\hline & & \multicolumn{2}{|c|}{ With ICM } & \multirow[t]{2}{*}{ Without ICM } & \\
\hline & & Incorporate & Corporate & & \\
\hline 1 & 29 & $11(37.9)$ & $10(34.5)^{a}$ & $5(17.2)$ & $3(10.3)$ \\
\hline 2 & 24 & - & $16(66.7)^{b}$ & $4(16.7)$ & $4(16.7)$ \\
\hline 3 & 25 & - & $20(80.0)^{\mathrm{c}}$ & $3(12.0)$ & $2(8.0)$ \\
\hline
\end{tabular}

Values within columns with different superscripts are significantly different $(\mathrm{a}-\mathrm{c}, \mathrm{P}<0.05)$

Method 1: Injection of the donor ICM, whereas the ICM of the recipient blastocyst is in 9 o'clock position, resulted in the two incorporated or corporated ICMs

Method 2: Injection of the donor ICM, whereas the ICM of the recipient blastocyst is in 3 o'clock position, resulted in the development of the donor ICM and broken recipient ICM

Method 3: Cutting the original ICM followed by the insertion of the donor ICM, resulted in the development of donor ICM with the origin recipient trophoblast 

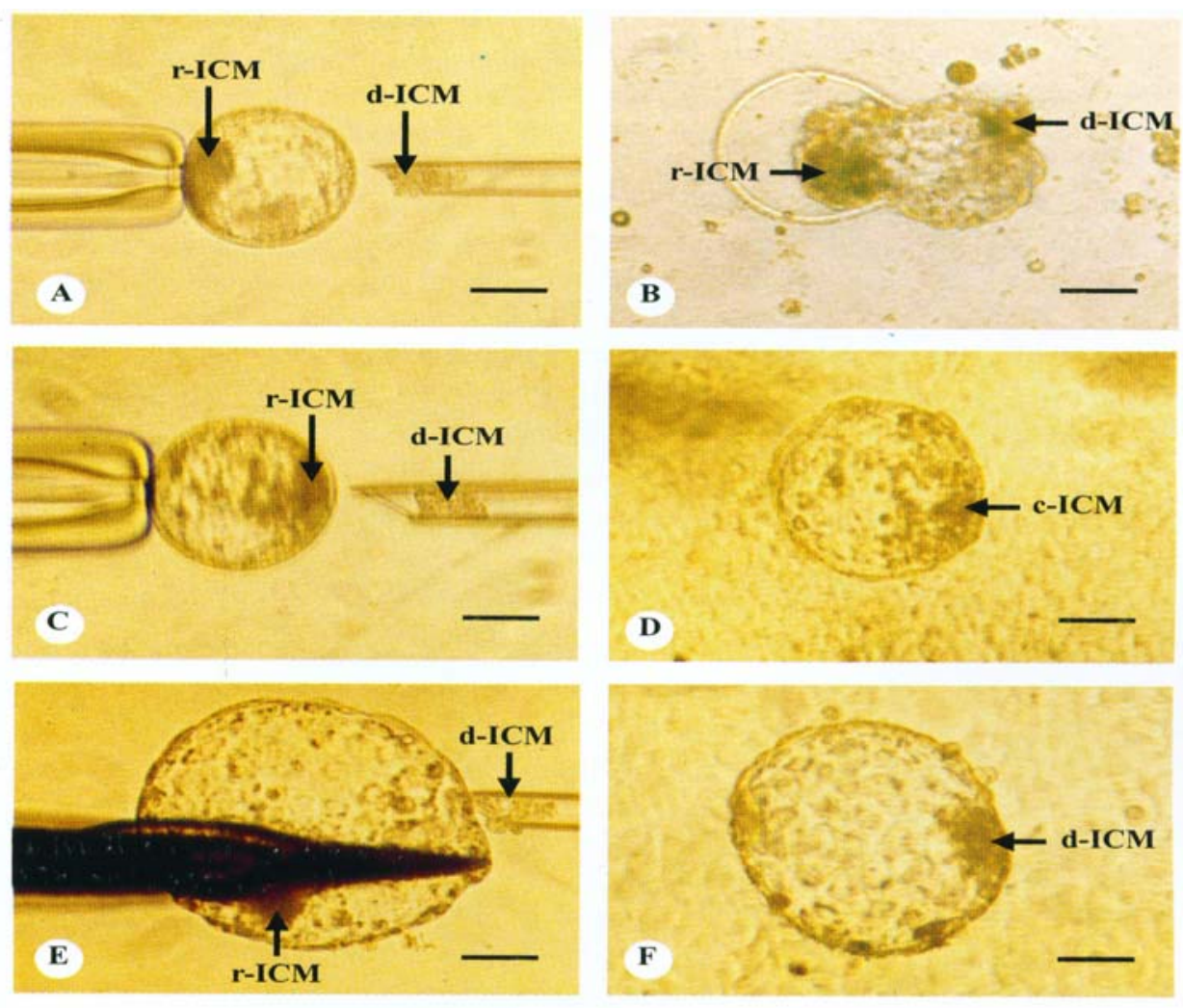

Figure 1 Production of the reconstructed embryo by transplantation of the donor ICM to the recipient bovine blastocyst. Method 1 (A and B): injection of the donor ICM, whereas the ICM of the recipient blastocyst in 9 o'clock position, resulted in the two incorporated ICMs. Method 2 (C and D): injection of the donor ICM, whereas the ICM of the recipient blastocyst is in 3 o'clock position, resulted in the development of the donor ICM and broken recipient ICM. Method 3 (E and F): cutting the original ICM followed by the insertion of the donor ICM, resulted in the development of donor ICM with the origin recipient trophoblast. ICM: inner cell mass, r-IMC: recipient ICM, d-ICM: donor ICM, c-ICM: corporate ICM. Bar $=50 \mu \mathrm{m}$.

species sheep-goat chimeras, produced one kid that developed from the donor ICM, two chimeras and 10 lambs after transfer of 22 reconstructed blastocysts into 14 recipients.

None of the reconstructed blastocyst produced by Method 2 developed with two incorporated ICM. A total of 66.7 percent of the reconstructed blastocysts developed with a single ICM. The purpose of this method was to break the original recipient ICM and insert the doonor ICM. However, we are not sure that the broken original ICM would be destroyed and the developed ICM come only from donor ICM. There was a possibility that the original ICM could recover and develop 
incorporating both ICMs from donor and recipient. The transfer of these embryos may result in chimeric animals.

To avoid the development of the original recipient ICM into the reconstructed blastocyst, the removal of ICM recipient blastocyst by cutting followed by insertion of the donor ICM (Method 3 ) is the most definite way to produce interspecies pregnancy. In this study, $80.0 \%$ of the reconstructed blastocyst developed with the ICM originally from donor ICM. The reconstructed blastocysts have been transferred to the three recipients resulting in one pregnancy. This method would be useful for production of the interspecies embryo transfer to rescue the endangered animals or for nuclear transfer produced embryo (Sansinena et al. 2005).

\section{CONCLUSIONS}

Attempts to produce interspecies embryos for transfer showed that the reconstructed blastocyst is better produced by cutting the original recipient ICM followed by the insertion of the donor ICM (Method 3 in this study) and transferring into recipient with the same species as recipient blastocyst.

\section{ACKNOWLEDGMENTS}

The author would like to thank Dr. Robert A. Godke, Professor of the Department of Animal Science, Louisiana State University, USA for his suggestions and helpful evaluation of the manuscript.

\section{REFERENCES}

Butler, J.E., Anderson, G.B., BonDurant, R.H., Pashen, R.L, and M.C. Penedo. 1987. Production of ovine chimeras by inner cell mass transplantation. J. Anim. Sci., 65:317-324. Boediono, A., Oe, M, Yamamoto, M,

Takagi, M., Saha, S. and T. Suzuki. 1993. Production of chimeric calves by aggregation of in vitro bovine embryos without zonae pellucidae. Theriogenology, 40:1221-1230. Boediono, A., Takagi, M., Saha, S. and S. Suzuki. 1994. The influence of day 0 and day 7 superovulated cow serum during development of bovine oocytes in vitro. Reprod. Fertil. Dev., 6:261-264. Boediono, A., Suzuki,

T., Li, L.Y. and R.A. Godke. 1999. Offspring born from chimeras reconstructed from parthenogenetic and in vitro fertilized bovine embryos. Mol. Reprod. Dev., 53:159-170. Boediono, A., Suzuki, T. and R.A. Godke. 2003. Comparison of hybrid and purebred in v/fro-derived cattle embryos during in vitro culture. Anim. Reprod. Sci.,78:l-l 1. Brackett, B.C. and G. Oliphant. 1975. Capacitation of rabbit spermatozoa in vitro. Biol. Reprod., 12. 260-274. Brem, G., Tenhumberg, H., and Karausslich. 1984. Chimerism in cattle through microsurgical aggregation of morula. Theriogenology, 22:609-613. Fehilly, C.B., Willadsen, S.M. and E.M. Tucker. 1984a.

Experimantal chimaerism in sheep. J. Reprod. and goat. Fertil., 70:347-351. Fehilly, C.B., Willadsen, S.M. and E.M. Tucker. 1984b. Interspecific chimerism between sheep

Nature, 307:634-636. Picard, L, Chartrain, I., King, W.A. and K.J. Betteridge. 1990. Production of chimaeric bovine embryos and calves by aggregation of inner cell masses with morulae. Mol. Reprod. Dev., 27:295-304. Polzin, V.J., Anderson, D.L., Anderson, G.B., BonDurant, R.H., Butler, J.E., Pashen, R.L., Penedo, M.C.T. and J.D. Rowe. 1987. Production of sheep-goat chimeras by inner cell mass transplantation. J. Anim. Sci.. 65:325-330. 
BIOTROPIA VOL. 13 NO. 2,2006

Rorie, R.W., Pool, S.H., Prichard, J.F., Betteridge, K.J. and R.A. Godke. 1994. A simplified procedure for making reconstructed blastocysts for interspecific and intergeneric transfer. Vet. Rec., 135:186-187. Sansinena, M.J., Hylan, D., Hebert, K., Denniston, R.S. and R.A. Godke. 2005. Banteng (Bos javanicus) embryos and pregnancies produced by interspecies nuclear transfer. Theriogenology, 63:1081-

1091. Solter, D. and B.B. Knowles. 1975. Immunosurgery of mouse blastocyst. Proc. Natl. Acad. Sci. USA., 72:5099-5102. 